\title{
On the nature of monster sawteeth during ICRF heating
}

\author{
S. Bernabei, R. Budny, E. D. Fredrickson, N. N. Gorelenkov*, J. C. Hosea, R. Majeski,
} C. K. Phillips, G. Schilling, J. R. Wilson.

Princeton Plasma Physics Laboratory, Princeton, N. J. 08543

*TRINITI, Troitsk, Moscow, Russia 142092

\begin{abstract}
A correlation between the presence of Energetic Particle Modes and long period sawteeth is explored. The crash of monster sawteeth is explained in terms of the loss of the stabilizing fast particles due to the EPM. High $\mathrm{q}_{\mathrm{a}}$ discharges, which never develop long period sawteeth, are explained in terms of ion loss due to Toroidal Alfvén Eigenmodes (TAE).
\end{abstract}

PACS numbers: 52.50.Gj, 52.55.Fa

In high power ICRF minority heating experiments a fast ion distribution is created in the plasma [1]. It has been observed that when this hot ion pressure is created inside of the radius where $\mathrm{q}=1, \mathrm{r}_{\mathrm{q}=1}$, it lengthens the sawtooth period as predicted theoretically [2,3]. In TFTR [4] and other machines it has been observed that the length of the sawtooth period varies at different plasma conditions. In specific cases the sawtooth can be lengthened by a factor of 15 or more. Very interestingly, long and ultra-long (monster) sawteeth can be observed in the same discharge (figure 1). The conditions favorable for monster sawteeth depend on the minority concentration, the ICRF resonance location and 
most interestingly the value of the cylindrical value of $q$ at the edge, $q_{a}$. Specifically, monster sawteeth are observed at $\mathrm{q}_{\mathrm{a}} \leq 3.6$, with a power threshold $\approx 2.8 \mathrm{MW}$. Figure 1 shows an example of the two kinds of sawteeth in the same discharge. Another phenomenon, observed in the same discharges with monster sawteeth, is the destabilization of Alfvén instabilities [5,6] (Fig. 2). In this Letter we discuss the correlation between these two phenomena. It is postulated here that the termination of the long sawtooth is due to depletion of stabilizing ions from the core of the plasma due to the Alfvén instabilities.

These experiments were conducted in ICRF-only, hydrogen minority, L-mode plasmas in the TFTR tokamak. The parameter ranges were: density $2-4.6 \times 10^{19} \mathrm{~m}^{-3}$, plasma current 1.2-1.8 MA, toroidal field 30-44.8 kG, frequencies 43,47 and $63.6 \mathrm{MHz}$ with power up to $11 \mathrm{MW}$. $\mathrm{q}_{\mathrm{a}}$ ranged from 2.8 to 6.8 .

It has been demonstrated that Alfvén instabilities are responsible for the observed degradation of the ICRF heating efficiency in these TFTR discharges [5]. There are basically two kinds of instabilities observed in the Alfvén frequency range [7]. One kind is readily identified as the Toroidal Alfvén Eigenmode (TAE), by the dependence of the frequency on density. The other has a rapidly changing frequency, i.e. the frequency chirps. These chirping modes are observed to trigger fast ion losses. Analysis with the NOVA-K code [8] fails to predict these chirping modes, but the non-perturbative code HINST [9] finds a branch of shear Alfvén waves corresponding to the Energetic Particle Modes (EPM) predicted by Zonca and Chen [10]. The analysis with HINST indicates that these modes should be localized to the core and reside in the Alfven continuum. HINST simulations also predict that as $\mathrm{q}_{0}$ decreases, due to the resistive current diffusion, the 
frequency of the modes should decrease (figure 3). In addition the HINST code predicts the destabilization of modes with a succession of toroidal number 'n' in decreasing order, as observed in the experiment and in agreement with theory [5,10]. More importantly HINST also predicts that the modes move radially outward in agreement with the experimental data [5] (figure 4). We carried out simulations with the ORBIT code [11] and found that the two conditions which lead to fast particle losses are that either the mode(s) is global, or it moves radially outward, transporting fast particles with it.

In previous experiments, loss of sawtooth stabilization was explained by widening of the $\mathrm{r}_{\mathrm{q}=1}$ radius until the hot ion beta is no longer sufficient for stabilization of the $\mathrm{m}=1$ mode. A puzzle in this explanation is that the stabilization occurs at low $\mathrm{q}_{\mathrm{a}}$ where the $\mathrm{r}_{\mathrm{q}=1}$ is fairly large and not at high $\mathrm{q}_{\mathrm{a}}$ when it is much smaller. At the same time it is also puzzling that the EPM appear earlier in time than the TAE, considering that the hot ion pressure gradient required is higher in the case of EPM (due to strong continuum damping).

The solution of these puzzles rests in the relative spatial location of the EPM and TAE. At low $\mathrm{q}_{\mathrm{a}}$ the structure of the Alfvén spectrum produces an Alfven frequency gap narrow enough to restrict the TAE to the outer half of the plasma, or more importantly away from the core where the fast ion distribution is formed. For the shot with the conditions of figure 1, the Alfven gap is shown in figure 5. The fast ion distribution in this case is calculated with the ICRF package of TRANSP [12]. The entire fast ion distribution is formed well inside the $\mathrm{r}_{\mathrm{q}=1}$, and is not in contact with the TAE (fig.6). This is a situation in which, aside from fishbone-like $\mathrm{n}=1$ burst modes, which are responsible for minor radial transport [5], no other loss mechanism is present to deplete the fast ion 
distribution. This allows the formation of a population of fast particles strong enough to stabilize the sawtooth for a long time. At the same time, the very same strong population can form a gradient large enough to destabilize EPM. As the EPM propagate radially outward, they carry fast particles with them. When the depletion of fast ions inside $r_{\mathrm{q}=1}$ becomes too severe, not enough fast ions are left to stabilize the sawtooth and a giant crash occurs.

Conversely, at high $\mathrm{q}_{\mathrm{a}}$, because of the steep gradient of the q-profile, the Alfvén gap widens enough to bring the TAE in contact with the fast ion population being created in the core (Figure 7). In this situation, as the fast ion population is formed by ICRF, it is readily depleted by the TAE. Therefore, a population of fast ions cannot be built up to the level required for sawtooth stabilization or for EPM destabilization.

Looking at figure 8, which shows the sawtooth period vs. RF power, it is clear that at low $\mathrm{q}_{\mathrm{a}}$ there are two groups of sawteeth, some which are lengthened by a factor of $\sim 4$ and others by a factor of $\sim 20$. There are no points in between, indicating a bifurcation. The scenario shown schematically in figure 9 could explain the situation. As time progresses two events happen in parallel: the hot ion pressure $\left(\mathrm{P}_{\text {hot }}\right)$ in the core starts increasing and the $\mathrm{q}_{0}$ decreases, widening $\mathrm{r}_{\mathrm{q}=1}$. It appears that at the beginning there is a period of marginal stability during which the few losses due to the Fishbone-like burst modes could cause a sawtooth crash. The heavy line represents the required $\mathrm{P}_{\text {hot }}$ for stabilization and the almost parallel line the increase of $\mathrm{P}_{\text {hot }}$ with ICRF heating. If the critical initial phase is passed, there is ample fast ion population for sawtooth stabilization. Previously it has been assumed that at this point the sawteeth are stable until $\mathrm{r}_{\mathrm{q}=1}$ widens sufficiently so that the fast ion population is no longer sufficient for 
stabilization. If this was the case the long period shown in fig. 8 should increase with power instead of being nearly independent of power.

Considering what has been shown in this paper, in scenarios involving high $1_{i}$ it is intriguing to speculate what would happen if the root of the problem, the decrease of $\mathrm{q}_{0}$ during ICRF, could be prevented. The EPM could still be destabilized, but would remain confined in the core and wouldn't initiate the loss which ultimately brings the sawtooth crash. This could conceivably be achieved with counter-current drive in the core, for example with ECCD.

This work supported by U. S. Department of Energy Contract No. DE-AC02-76CHO-3073.

\section{REFERENCES.}

[1] J. R. Wilson,M. G. Bell, H. Biglari, et al., in Plasma Physics and Controlled Nuclear Fusion Research 1992 (Proc. 14th Int. Conf. Würzburg, 1992), vol.1 (International Atomic Energy Agency, Vienna 1993) 661.

[2] D. J. Campbell, D. F. Start, J. A. Wesson et al., Phys. Rev. Lett. 60, 2148 (1988).

[3] R. B. White, E. Fredrickson, D. Darrow et al., Phys. Plasmas 2, (1995) 2871.

[4] R. Hawryluk, V. Arunasalam, C. W. Barnes, et al., Plasma Phys. Controlled Fusion 33, (1991) 1509 .

[5] S. Bernabei, M. G. Bell, R. Budny et al., Phys. Of Plasmas 6, no. 5, (1999) 1880.

[6] L.-G. Ericksson, M. Mantsinen, D. Borba et al., Phys. Rev. Lett . 81, no. 6 (1998), 1231.

[7] E. D. Fredrickson, M. G. Bell, R. V. Budny et al., Nucl. Fus. 35, (1995) 1457.

[8] G. Y. Fu, C. Z. Cheng, R. Budny et al., Phys. Plasmas 3, (1996) 4036. 
[9] N. N. Gorelenkov, C. Z. Chang and W. M. Tang, Phys. Plasmas 5, (1998) 3389.

[10] F. Zonca and L. Chen, Phys. Plasmas 3, (1996) 323.

[11] R. B. White and M. S. Chance, Phys. Fluids 27, 2455 (1984).

[12] R. V. Budny, M. G. Bell, A. C. Janos et al., Nucl. Fus. 35, (1995) 1497. 


\section{FIGURE CAPTIONS.}

Fig. 1 X-ray signal showing two monster sawteeth and a shorter one; the last sawtooth is due to the RF power termination. $\mathrm{I}_{\mathrm{p}}=1.68 \mathrm{MA}, \mathrm{B}_{\mathrm{T}}=30 \mathrm{kG}, \mathrm{q}_{\mathrm{a}}=3.2$.

Fig. 2 TAE and EPM from a magnetic probe, preceding a monster sawtooth. $\mathrm{q}_{\mathrm{a}}=3.2$, $\mathrm{P}_{\mathrm{RF}}=5.2 \mathrm{MW}$

Fig. 3 Variation of the EPM frequency, normalized to the value of the central Alfvén frequency at $\mathrm{q}_{\mathrm{o}}=0.9$, vs. decreasing $\mathrm{q}_{\mathrm{o}}$.

Fig. 4 Variation of the EPM frequency, normalized to the central Alfvén frequency at $\mathrm{q}_{\mathrm{o}}=0.9$, as the modes move outwards.

Fig. 5 Alfvén frequency gap calculated by NOVA-K for a discharge with $\mathrm{q}_{\mathrm{a}}=3.2$

Fig. 6 TRANSP calculation of the q-profile and of the hot ion pressure generated by ICRF heating. $\mathrm{q}_{\mathrm{a}}=3.2, \mathrm{P}_{\mathrm{RF}}=6.2 \mathrm{MW}$.

Fig. 7 Alfvén frequency gap calculated by NOVA-K for a discharge with $\mathrm{q}_{\mathrm{a}}=4.5$

Fig. 8 Sawtooth period versus RF power. Full circles are at $\mathrm{q}_{\mathrm{a}}=3.2$, open circles at $\mathrm{q}_{\mathrm{a}}=4.5$ 
Fig. 9 Scenario showing that $\mathrm{P}_{\text {hot }}$ generated by ICRF heating initially approaches the value required for stabilization of the sawtooth, then grows faster providing a good margin for stability until its growth is clamped by the onset of EPM. Shorter sawtooth refers to the lower points in fig.8 


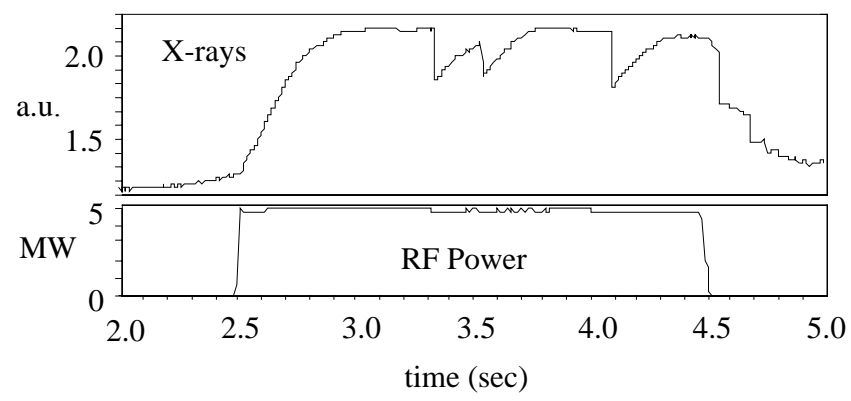

S. Bernabei / P.R.L. Fig. 1 


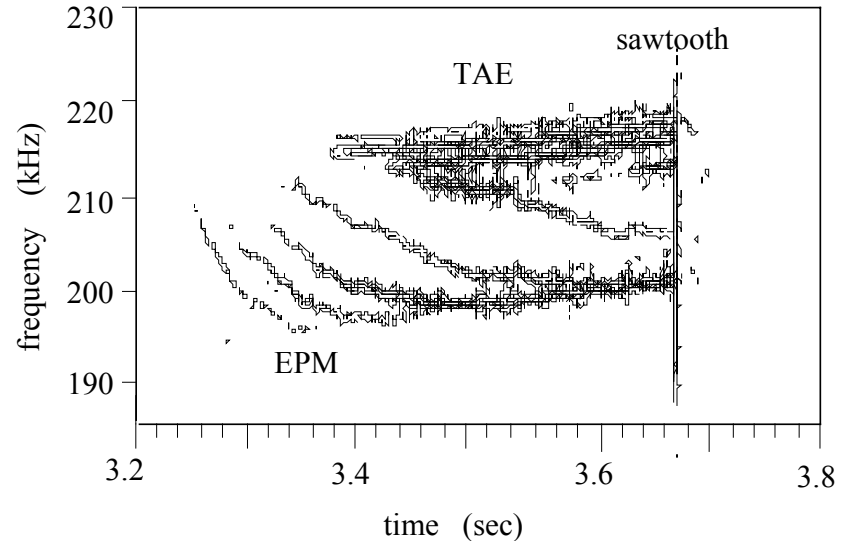

S. Bernabei / P.R.L. Fig. 2 


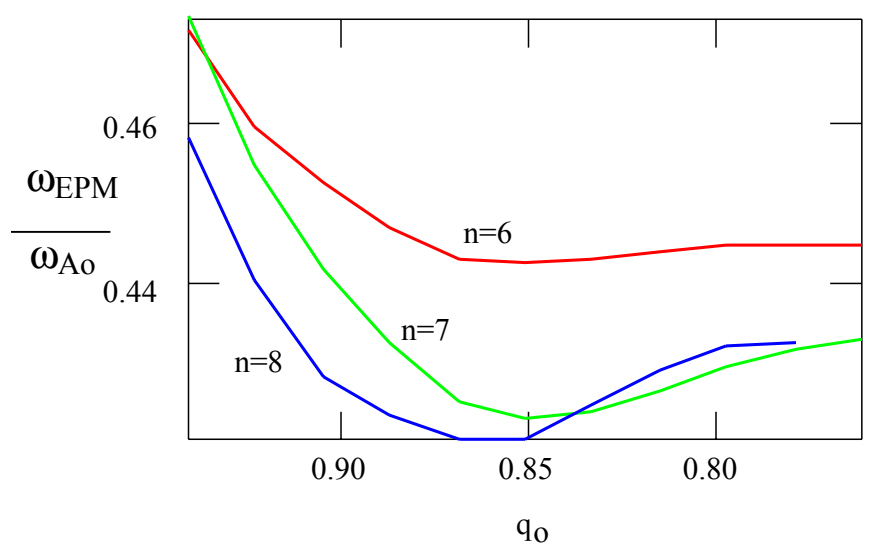

S. Bernabei / P.R.L. Fig. 3 


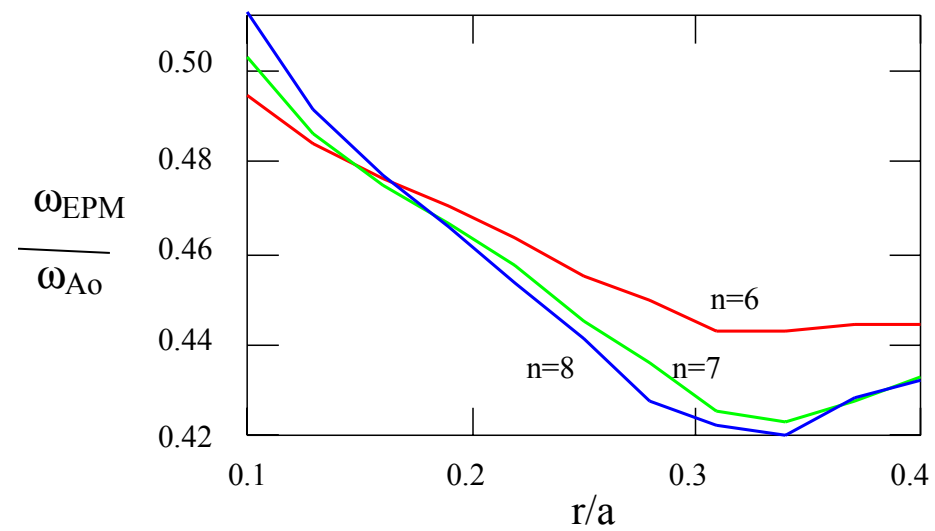

S. Bernabei / P.R.L. Fig. 4 


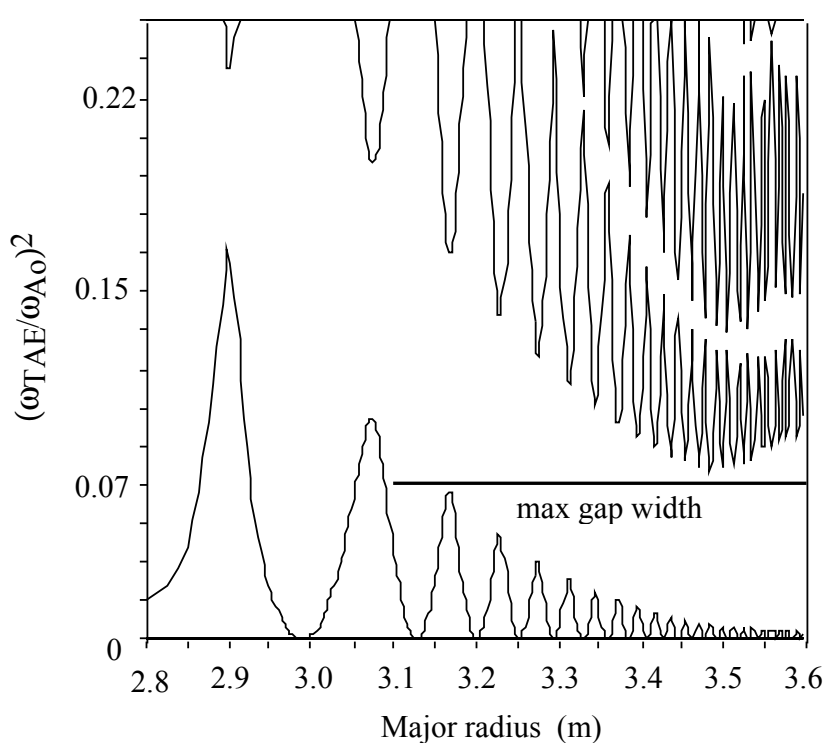

S. Bernabei / P.R.L. Fig. 5 


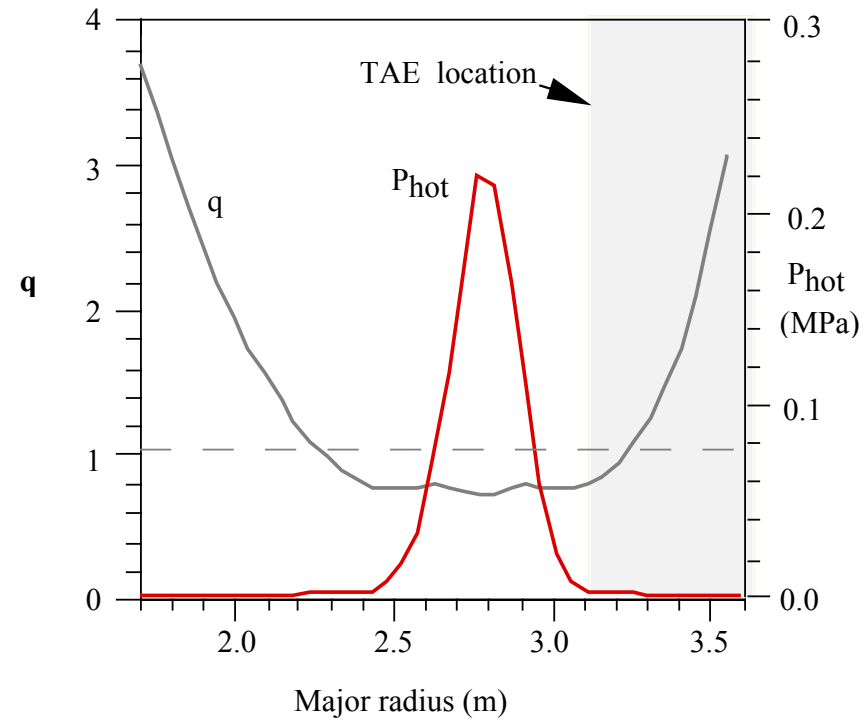

S. Bernabei / P.R.L. Fig. 6 


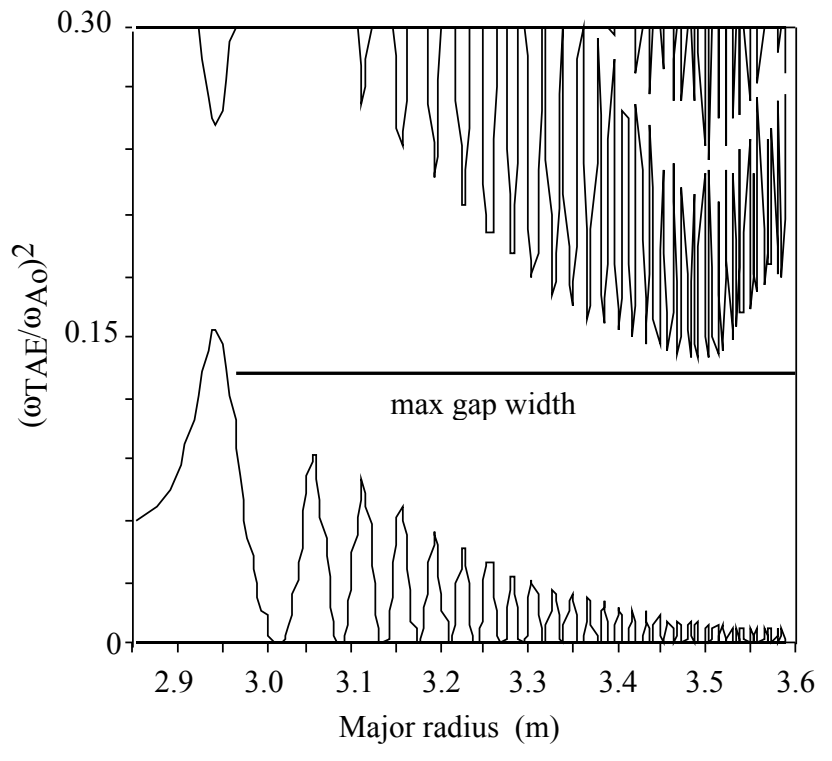

S. Bernabei / P.R.L. Fig. 7 


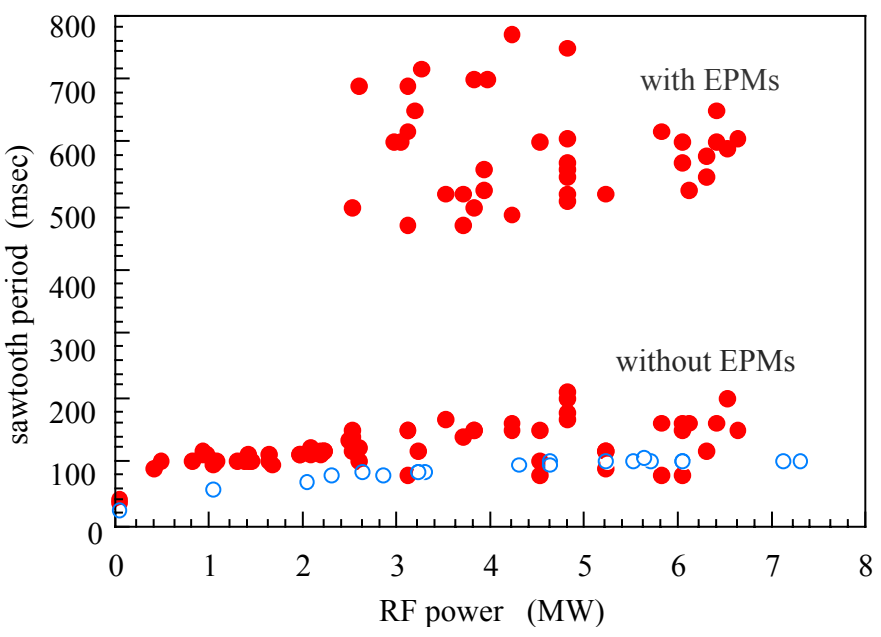

S. Bernabei / P.R.L. Fig. 8 


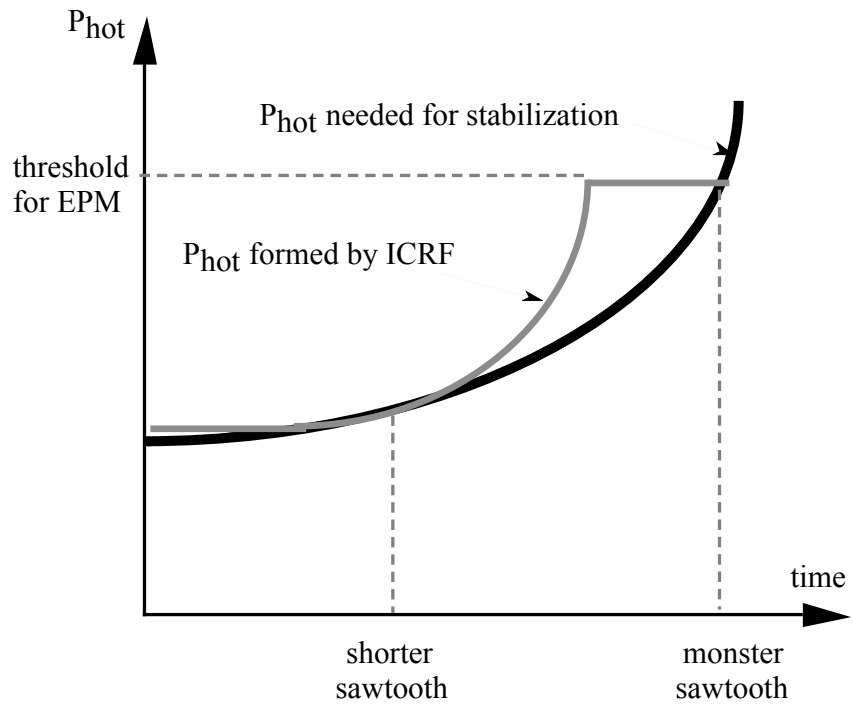

S. Bernabei / P.R.L. Fig. 9 